\title{
An Improved Analytical Method for Dimensioning Wireless Multihop Networks Jointly Combining Interference and Traffic Effects
}

\author{
Renata Valverde Mello, Flávio du Pin Calmon, Álvaro Augusto Machado de Medeiros and Michel Daoud Yacoub
}

\begin{abstract}
Resumo-O dimensionamento de redes multihop sem fio é uma tarefa complexa que pode ser abordada de diversas maneiras, mais notoriamente por meio de simulações ou pela análise de limites teóricos. Neste artigo, é proposto um novo método analítico para o dimensionamento de redes multihop sem fio, considerando conjuntamente as limitações de (i) recursos e (ii) interferência, impostas por efeitos do tráfego e do canal combinados. Este método é baseado na formulação da probabilidade de outage da rede, i.e., a probabilidade de que a transmissão entre dois nós falhe devido à falta de recursos ou porque a razão sinal-interferência-mais-ruído está abaixo de determinado limiar. $O$ método analítico ainda contempla o efeito conjunto do tráfego multihop e seu consequente aumento na interferência, considerando os efeitos do ruído aditivo gaussiano, da interferência mútua e do roteamento. As formulações propostas são ilustradas com exemplos analíticos e validadas por meio de simulação.
\end{abstract}

Palavras-Chave-Redes Multihop sem fio, Probabilidade de Outage , Razão Sinal-Interferência-mais-Ruído, Rayleigh

Abstract-Dimensioning wireless multihop networks is a complex task that has been tackled through different approaches, most notably simulation or analysis of theoretical bounds. In this paper, we propose a novel analytical method for dimensioning wireless multihop networks, considering both (i) resource and (ii) interference limitations imposed by traffic and channel effects combined. This method is based on the formulation of the outage probability of the network, i.e., the probability that a transmission between two nodes fails due to lack of resources or because the signal-to-interference-plus-noise ratio is below a specific threshold. The analytical method also encompasses the joint effect of the multihop traffic and its consequent interference increase, considering the effects of additive noise, mutual interference and routing. The proposed formulations are illustrated with practical examples and validated through simulation.

Keywords - Wireless Multihop Networks, Outage Probability, Signal-To-Interference-Plus-Noise Ratio, Rayleigh

\section{INTRODUCTION}

Wireless multihop networks are composed of nodes that may transmit, receive or route information using a wireless

Renata Valverde Mello, Flávio du Pin Calmon, and Michel Daoud Yacoub are with the Wireless Technology Laboratory (WissTek), Department of Communications, School of Electrical and Computer Engineering, University of Campinas, PO Box 6101, 13083-852, Campinas, SP, Brazil (E- mail: [renata,fcalmon,michel]@wisstek.org).

Álvaro Augusto Machado de Medeiros is with the CPqD Foundation, Rod. SP-340 - km 118.5, 13086-902, Campinas, SP, Brazil (E- mail: amachado@cpqd.com.br).

This work was partly supported by FAPESP (07/02117-1) and by CAPES. medium. From fixed wireless mesh networks to mobile ad hoc networks, the advent of multihop transmissions is becoming a established technique for future wireless networks. However, dimensioning wireless multihop networks, i.e. determining the number of users and their respective data rates, is not a simple task. The wireless medium adds randomness to the problem because transmissions may or may not interfere with each other depending on the channel conditions, which degrades the quality of service (QoS). In addition, the multihop traffic intensifies the dependence among nodes, increasing the network traffic, hence the number of blocked transmissions, which, in turn, deteriorates the grade of service (GoS).

The dimensioning of wireless ad hoc networks has been tackled in the literature through various approaches, including capacity analysis through simulation [1] and upper and lower bounds determination [2]. Closed-form expressions for the blocking probability, in a linear topology wireless multihop network considering interference without random fading, have been derived and many analytical and semi-analytical methods for calculating the blocking probability have been proposed [3]. Nevertheless, those methods do not consider the multihop traffic and the fading drawbacks [3] nor the interference effects combined [4].

Furthermore, an analytical dimensioning method based on the outage probability, which is a more appropriate performance measure, was introduced in [5]. Here, the effect of interference is considered when admitting a call to the system, verifying if the signal-to-interference ratio (SIR) threshold required by the new call is attended, given the number of interferers and the channel condition. However, this is an approximation, since it analyzes only the signal-to-interference ratio along the route of the new call, not considering the effect of the interference generated by the new call on the ongoing transmissions. To overcome this limitation, i.e., to account for the effect of the admitted call on the system as a whole, it is necessary to apply the concept of joint outage probability (JOP) [6].

In light of the previous discussion, this paper extends this analytical method in order to encompass multihop traffic, additive noise, mutual interference and routing effects combined, resulting in a more complete and realistic formulation. Furthermore, these results are applied in a practical scenario of wireless multihop network dimensioning and validated through simulation. Additionally, the exact expression for Joint Outage Probability with additive noise 
is derived.

The remainder of this paper is structured as follows. Section II defines the network characteristics. Section III describes the method and its extension to support multiple routes. Section IV describes the interference model and introduces the expressions for the JOP considering additive noise. Section V compares our solution with simulated values. Finally, Section VI presents some concluding remarks and a summary of the results.

\section{NeTwORK MODEL}

Consider a network with $N$ nodes connected through $L$ links. Each link $l, l=1, \ldots, L$, connects two nodes and has a capacity of $C_{l}$ trunks (interchangeably used with circuits or units of bandwidth). Each trunk supports a call or data flow between two nodes, and calls within the given link are separated by a code, as in Direct Sequence Code Division Multiple Access (DS-CDMA). For the sake of simplicity, links are assumed not to interfere with each other, but calls within the same link, which share the same frequency, can interfere with each other if the signal-to-interference-plus-noise ratio exceeds a certain threshold $\beta$. To encompass the case with interference from other links, the same analysis made in [5] can be applied. by

The signal-to-interference-plus-noise ratio $E_{b} / I_{N}$ is given

$$
\frac{E_{b}}{I_{N}}=\frac{G W_{j}}{\sum_{i=1}^{C_{l}} W_{i}+n_{j}}
$$

where $G$ is the processing gain, $W_{j}$ is the received power from the desired signal, $W_{i}$ is received power from interferer $i$, and $n_{j}$ is the additive white Gaussian noise.

The topology of the network is arbitrary, with no specific constraints. A source-destination node pair $(x, y)$ is connected through routes composed of links between them. When the communication between a source-destination node pair is established, each link on route $r$ has an occupied trunk. If at least one of the links in a route has no free trunk, out of all $C_{l}$ ones, the call is blocked. If the call is not blocked, but the interference $I_{N}$ on any link of its route increases, so that $E_{b} / I_{N}$ is below a given threshold for this new call and for all existent calls, then this call is not admitted. The probability of the denial of service, in any one of these cases, is defined as the outage probability.

In our approach, we assume the following:

1) The calls arrive at each source-destination pair of nodes $(x, y)$ as a Poisson process with rate $\lambda_{x, y}$ and has a probability of selecting one of the routes connecting this pair. Furthermore, and the call service time has an exponential distribuition with its mean time designated by $1 / \mu$. The total load offered to an individual link is also a Poisson process with rate influenced by the outage condition of other links.

2) Outage occurs independently, from link to link, determined by their respective arrival rates. That is, even though a route is composed by successive links creating

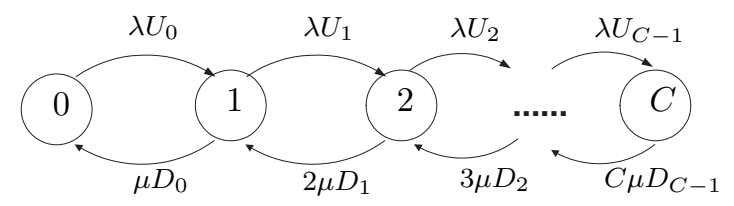

Fig. 1. State diagram for a single link with interference on call admission and call maintenance

dependence among them (and so is the outage on these links), we will, nevertheless, treat them as independent.

3) All links are assumed non-directional. For traffic between two nodes, there is no difference between source and destination. This assumption is adopted only for simplicity of notation. Our model can also be applied to directional link scenarios.

4) The powers $W_{j}$ and $W_{i}$ are modeled as exponential random variables, i.e. the nodes transmissions are affected by the Rayleigh fading. Others types of fading can also be adopted.

5) All links in the network have fixed capacity. Reduction in capacity or unavailability of link occurs only due to interference generated by other calls or occupation of trunks. This assumption restricts the mobility of nodes, but it can be considered through simulation [4].

6) All nodes transmit at the same power, although our model can also be applied with nodes having distinct transmission powers.

\section{Analytical Method}

We define the traffic or offered load $A$ as $A=\lambda / \mu$. From the Markov chain depicted in Figure 1, the derivation of the state probability $S_{k}$, i.e. the probability of $k$ occupied trunks, is obtained as

$$
S_{k}=\frac{\frac{A^{k}}{k !} \prod_{i=0}^{k-1} \frac{H_{i}}{D_{i}}}{1+\sum_{m=1}^{C} \frac{A^{m}}{m !} \prod_{i=0}^{m-1} \frac{H_{i}}{D_{i}}} .
$$

As in [5], we define $H_{i}$ as a function affecting the call arrival rate and a function $D_{i}$ that affects the call departure time. Both functions are here modeled by the Joint Outage Probability, which is described in Section IV, such as

$$
\begin{aligned}
H_{k} & =P_{\bar{I}}\left(Z_{\lambda}, \Omega, \sigma^{2}, k\right), \\
D_{k} & =1 / P_{\bar{I}}\left(Z_{\mu}, \Omega, \sigma^{2}, k\right),
\end{aligned}
$$

where $\Omega$ is the transmission power and $\sigma^{2}$ is the variance of the additive white Gaussian noise, $Z_{\lambda}$ and $Z_{\mu}$ denote the respective interference thresholds. The interference threshold is here defined as $G / \beta$. Different values for $Z_{\lambda}$ and $Z_{\mu}$ may be used in order to have a hysteresis between admission and dropping to prevent the ping-pong (occupation) effect.

The mean number of occupied trunks for a link with $C$ trunks is 


$$
E_{C}[k]=\sum_{k=1}^{C} k S_{k}
$$

Following the classical definition of carried load, we use an expression proposed in [5], that associates the new carried load with the mean number of occupied trunks, i.e. $A\left(1-O_{C}\right)=$ $E_{C}[k]$, where $O_{C}$ is the outage probability of a link with $C$ trunks. Thus,

$$
O_{C}=1-\frac{E_{C}[k]}{A}
$$

Therefore, to calculate the outage probability of a link $l$, the entries are the load offered to this link $A_{l}$, the link's capacity $C_{l}$ and the interference margins $Z_{\lambda}$ and $Z_{\mu}$. We refer to this method as

$$
O_{l}=\operatorname{Out}\left(A_{l}, C_{l}, Z_{\lambda}, Z_{\mu}\right) \text {. }
$$

We define the array $R_{x, y}=\left\{r_{x, y}^{1}, r_{x, y}^{2}, \ldots, r_{x, y}^{R}\right\}$ as the set of routes that connects a pair of nodes $(x, y)$. Each route has a probability of being selected, determined by a routing algorithm, such that $\rho_{x, y}=\left\{q_{x, y}^{1}, q_{x, y}^{2}, \ldots, q_{x, y}^{R}\right\}$ is the associated probability array of $R_{x, y}$. Thus, we have that $\sum_{r=1}^{R} q_{x, y}^{r}=1$.

The array $\rho_{x, y}$ can be used on the reduced load approximation proposed in [5] to encompass multiple routes. Assuming that the outage occurs independently from link to link, the connections arrive at link $l$ according to a Poisson process with offered load

$$
A_{l}=\sum_{r \in R_{l}} A_{x, y} q_{x, y}^{r} \prod_{\substack{g \in r \\ g \neq l}}\left(1-O_{g}\right)
$$

where $R_{l}$ is the set of routes through link $l$ and $A_{x, y}$ is the traffic offered to each source-destination pair $(x, y)$.

Then, (7) is applied in (6) in order to find the outage probability of each link, as

$$
O_{l}=\operatorname{Out}\left(\sum_{r \in R_{l}} A_{r} q_{x, y}^{r} \prod_{\substack{g \in r \\ g \neq l}}\left(1-O_{g}\right), C_{l}, Z_{\lambda}, Z_{\mu}\right) .
$$

For the case of modeling admission control, only interference on arrival is considered and $Z_{\mu}=\infty$.

Assuming independence among links, the outage probability for each pair of nodes $O_{x, y}$

$$
O_{x, y}=1-\sum_{r=1}^{R_{x, y}} q_{x, y}^{r} \prod_{l \in r}\left(1-O_{l}\right) .
$$

Note that (8) is a transcendental equation that must be solved iteratively until the solution converges to a certain accuracy, established as desired. A possible way to solve this equation is through the relaxation method, in which an initial arbitrary input for an equation is given. As discussed in [7], this method converges to a unique point. That is why this method is also called fixed-point approximation.

\section{Joint Outage Probability With ADditive NOISE}

In order to model the effect of mutual interference in the proposed scheme, the Joint Outage Probability (JOP) of the calls will be considered. The JOP corresponds to the probability that, given a set of signal-to-interference-plus-noise ratio (SINR) restrictions for a group of mutually interfering signals, at least one signal will be in outage $\left(P_{I}\right)$, or, dually, all SINR restrictions will be attended $\left(P_{\bar{I}}\right)^{1}$. In interference-limited systems, i.e, systems where additive noise can be neglected, an exact formulation for the JOP is given in [6]. In the following, such previous formulation will be extended in order to encompass additive noise.

\section{A. Outline of the Problem}

Let $W_{i}, i=1, \ldots, N$, be the instantaneous powers of mutually interfering calls. As discussed, in order for a call not to be dropped, it is required that the signal-to-interference-plus-noise ratio at the receiver to be greater than a tolerable threshold $\beta_{i}, i=1, \ldots, N$, as specified for the particular communication. Each call is also subject to additive noise, with instantaneous power denoted by $n_{i}$. We consider, without loss of generality, that $n_{i}$ is constant. Therefore, the $N$ received signals must satisfy the set of inequalities

$$
\mathcal{S}_{\sigma}^{N}=\left\{\frac{W_{j}}{\sum_{\substack{i=1 \\ i \neq j}}^{N} W_{i}+n_{j}} \geq \beta_{j}, \quad j=1, \ldots, N\right.
$$

The inequalities in (10) describe a hypervolume in the $N$-dimensional space, represented by $\mathcal{S}_{\sigma}^{N}$. The Joint Outage Probability can be obtained as

$$
P_{\bar{I}}=\int_{\mathcal{S}_{\sigma}^{N}} f_{\mathbf{W}}\left(w_{1}, \ldots, w_{N}\right) d w_{1} \ldots d w_{N},
$$

where $f_{\mathbf{W}}\left(w_{1}, \ldots, w_{N}\right)$ is the joint probability density function of $W_{1}, \ldots, W_{N}$. In case the additive noise $n_{i}$ are also random variables, $N$ further integrations must be performed in order to obtain the exact value of $P_{\bar{I}}$.

\section{B. An Exact Solution for the JOP with Additive Noise}

An integral-form solution for the JOP when $n_{i}=0$ for all $i$, is given in [6], as well as a closed-form expression for an independent Rayleigh fading environment. With these results in hand, we will now proceed to reformulate the set of inequalities (10) into the region defined as $\mathcal{S}^{N}$ in [6, Eq. (1)], so that the previous results can be directly applied. For notation purposes, we define:

$$
a_{i}=\frac{\beta_{i}}{1+\beta_{i}} .
$$

Performing the change of variables

$$
W_{j}=U_{j}+g_{j}(\mathbf{a}, \boldsymbol{n})
$$

\footnotetext{
${ }^{1}$ In this article, the term JOP is used to denote $P_{I}$ as well as $P_{\bar{I}}$, since $P_{I}=1-P_{\bar{I}}$. The exact meaning will be clear from the context.
} 
where

$$
g_{j}(\mathbf{a}, \boldsymbol{n}) \triangleq a_{j}\left(\frac{\sum_{i=1}^{N} a_{i} n_{i}}{1-\sum_{i=1}^{N} a_{i}}+n_{j}\right)
$$

$\mathbf{a} \triangleq\left(a_{1}, a_{2}, \ldots, a_{N}\right)$ and $\boldsymbol{n} \triangleq\left(n_{1}, n_{2}, \ldots, n_{N}\right)$, and substituting (13) in (10), the set of signal-to-interference-plus-noise ratios can be rewritten as

$$
\left\{\frac{U_{j}}{\sum_{\substack{i=1 \\ i \neq j}}^{N} U_{i}} \geq \beta_{j}, \quad j=1, \ldots, N .\right.
$$

Note that the set of inequalities (15) is equivalent to the region $\mathcal{S}^{N}$ in [6]. Therefore, (11) can be expressed as

$$
\begin{gathered}
P_{\bar{I}}=\sum_{j=1}^{N-1} \int_{0}^{\infty} \int_{\mathbb{K}_{1, j} u_{j}}^{\left(\mathbb{C}_{j, N}-\sum_{\substack{i=2 \\
i \neq j}}^{N-1} \mathbb{K}_{i, j}\right) u_{j}} \ldots \\
\times \int_{\mathbb{K}_{j-1, j} u_{j}}^{\left(\mathbb{C}_{j, N}-\sum_{i=j+1}^{N-1} \mathbb{K}_{i, j}\right) u_{j}-\sum_{i=1}^{j-2} u_{i}} \int_{\mathbb{K}_{j+1, j} u_{j}}^{\left(\mathbb{C}_{j, N}-\sum_{i=j+2}^{N-1} \mathbb{K}_{i, j}\right) u_{j}-\sum_{i=1}^{j-1} u_{i}} \ldots \\
\times \int_{\mathbb{K}_{N-1, j} u_{j}} \int_{\beta_{N} \sum_{\substack{i=1 \\
i \neq j}}^{N-1} u_{i}}^{\mathbb{C}_{j, N} u_{j}-\sum_{\mathbf{i}}^{N-2} u_{i}} f_{\mathbf{W}}\left(u_{1}+b_{1}, \ldots, u_{N}+b_{N}\right) \\
\times d u_{N} d u_{N-1} \ldots d u_{j+1} d u_{j-1} \ldots d u_{1} d u_{j}, \quad(16)
\end{gathered}
$$

where $b_{i}=g_{i}(\mathbf{a}, \boldsymbol{n})$,

$$
\mathbb{K}_{j, k}=\frac{\beta_{j}\left(1+\beta_{k}\right)}{\beta_{k}\left(1+\beta_{j}\right)} \text { and } \mathbb{C}_{j, N}=\frac{\left(1-\beta_{j} \beta_{N}\right)}{\beta_{j}\left(1+\beta_{N}\right)} .
$$

\section{JOP for a Rayleigh Environment with Additive Noise}

Consider that the mutually interfering signals $W_{i}$ are independent, and each signal has a Rayleigh distribution with mean power $\Omega_{i}$. In this scenario, the result of the integration in (16) will be

$$
P_{\bar{I}}=P_{\bar{I} 0}\left(\boldsymbol{\Omega}, \mathbf{a}_{\mathbf{i}}\right) \exp \left(-\sum_{i=1}^{N} \frac{g_{i}(\mathbf{a}, \boldsymbol{n})}{\Omega_{i}}\right),
$$

where $\Omega \triangleq\left(\Omega_{1}, \ldots, \Omega_{N}\right)$. The function $P_{\bar{I} 0}\left(\boldsymbol{\Omega}, \mathbf{a}_{\mathbf{i}}\right)$ is the probability that all the inequalities in (10) are attended when $n_{i}=0$ for all $i$, given by [6]:

$$
P_{\bar{I} 0}\left(\boldsymbol{\Omega}, \mathbf{a}_{\mathbf{i}}\right)=\frac{\left(1-\sum_{j=1}^{N} a_{j}\right)^{N-1}}{\prod_{i=1}^{N}\left(1-\sum_{j=1}^{N} a_{j}\left(1-\frac{\Omega_{i}}{\Omega_{j}}\right)\right)} .
$$

If the additive noise $n_{i}$ are independent random variables, it is straightforward to show that the JOP in the independent

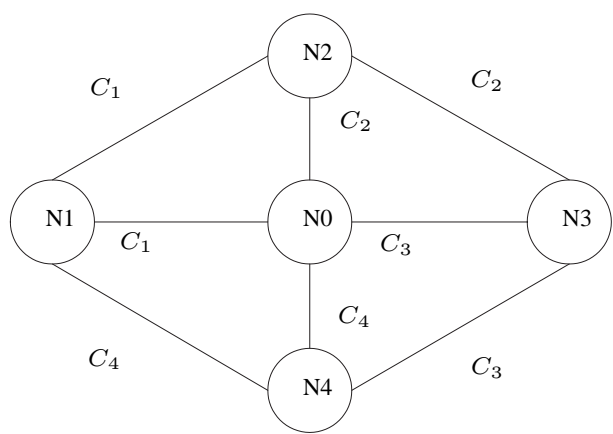

Fig. 2. An example of star network.

Rayleigh fading environment is

$$
P_{\bar{I}}=P_{\bar{I} 0}\left(\mathbf{\Omega}, \mathbf{a}_{\mathbf{i}}\right) \prod_{i=1}^{N} M_{n_{i}}\left[-g_{i}\left(\mathbf{a}, \mathbf{\Omega}^{-1}\right)\right],
$$

where $\Omega^{-1} \triangleq\left(\Omega_{1}^{-1}, \Omega_{2}^{-1}, \ldots, \Omega_{N}^{-1}\right), g_{i}(\cdot)$ is defined in (14) and $M_{n_{i}}[\cdot]$ is the Moment Generating Function of $n_{i}$.

When the additive noise has a Gaussian distribution with variance $\sigma^{2}$ and zero mean, $i=1, \ldots, N$, (19) becomes

$$
P_{\bar{I}}=P_{\bar{I} 0}\left(\boldsymbol{\Omega}, \mathbf{a}_{\mathbf{i}}\right) \prod_{i=1}^{N}\left(1+\sigma_{i}^{2} g_{i}\left(\mathbf{a}, \boldsymbol{\Omega}^{-1}\right)\right)^{-1 / 2}
$$

Considering a symmetric environment, i.e., $\beta_{i}=\beta, \Omega_{i}=\Omega$ and $\sigma_{i}^{2}=\sigma^{2}$ for all $i,(20)$ simplifies to

$$
P_{\bar{I}}\left(\beta, \Omega, \sigma^{2}, N\right)=\frac{\left(1-\frac{N \beta}{1+\beta}\right)^{3 N / 2-1}}{\left(1+\frac{\beta}{1+\beta}\left(\frac{\sigma^{2}}{\Omega}-N\right)\right)^{N / 2}}
$$

\section{An Application ExAmple}

In order to illustrate the proposed formulations, a multihop network with the topology depicted in Fig. 2 was analyzed. We consider that the calls arrive as a Poisson process for each pre-defined route. The routes are specified for each source-destination node pair as shown in Table I. All nodes but $N_{0}$, which is a realy node, generate and receive traffic. Only routes with two or less links are considered and, therefore, only 14 routes are defined for this scenario, with the probabilities of using one-link routes being higher than using two-link routes.

In this example, an admission control scenario is simulated, in which the power of the signal corresponding to a given user is exponentially distributed with average power $\Omega=1$ (Rayleigh fading). In addition, we considered that all the calls in the system were subject to additive Gaussian noise with zero mean and variance $\sigma^{2}=0.1$ (the exact power units are irrelevant, since we considered the ratio $\sigma^{2} / \Omega$ ). Before call acceptance, the following tests are performed. First, trunk availability is checked. In case the resource is available, the effect on the signal-to-interference-plus-noise ratio for all ongoing calls, as well as for the one in admission is checked 
XXVII SIMPÓSIO BRASILEIRO DE TELECOMUNICAÇÕES - SBrT 2009, DE 29 DE SETEMBRO A 2 DE OUTUBRO DE 2009, BLUMENAU, SC

TABLE I

DEFINED ROUTES FOR THE NETWORK DEPICTED IN FIGURE 2 AND ITS PROBABILITIES.

\begin{tabular}{ccc}
\hline Pair & Route & Route Probability \\
\hline \hline N1-N2 & N1-N2 & 0.6 \\
& N1-N0-N2 & 0.4 \\
\hline N1-N33 & N1-N0-N3 & 0.333 \\
& N1-N2-N3 & 0.333 \\
& N1-N4-N3 & 0.334 \\
\hline N1-N4 & N1-N4 & 0.6 \\
& N1-N0-N4 & 0.4 \\
\hline N2-N3 & N2-N3 & 0.6 \\
& N2-N0-N3 & 0.4 \\
\hline N2-N4 & N2-N0-N4 & 0.33 \\
& N2-N1-N4 & 0.33 \\
& N2-N3-N4 & 0.34 \\
\hline N3-N4 & N3-N4 & 0.6 \\
& N3-N0-N4 & 0.4 \\
\hline
\end{tabular}

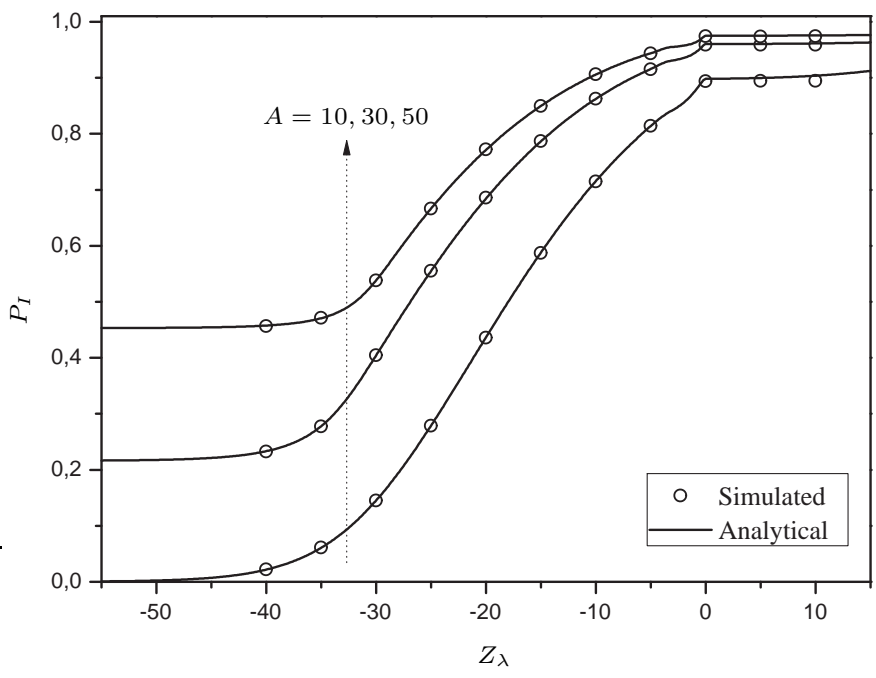

Fig. 3. Outage probability as a function of the SINR threshold $Z_{\lambda}$ considering two possible routes between source and destination nodes.

against the tolerable threshold. If this test passes, then the call is admitted. If any of the tests fail, the call is rejected.

The theoretical results for this scenario, along with simulated points, are presented in Fig. 3 and Fig. 4. The outage probability is represented in terms of the SINR threshold $Z_{\lambda}$ with $C 1=C 2=C 3=C 4=30$ and different traffic intensities. As can be observed, there is an excellent agreement between simulated and theoretical results.

Analyzing the results, we note that, for a small SINR threshold, interference will have little influence on the outage probability, and the system becomes limited by resource availability (traffic). By increasing $Z_{\lambda}$, the effect of interference among the calls becomes more noticeable, and, consequently, the outage probability steadily increases. When $Z_{\lambda} \geq 0 d \mathrm{~B}$, the system outage presents a saturation due to interference, since at most one network link can be occupied at

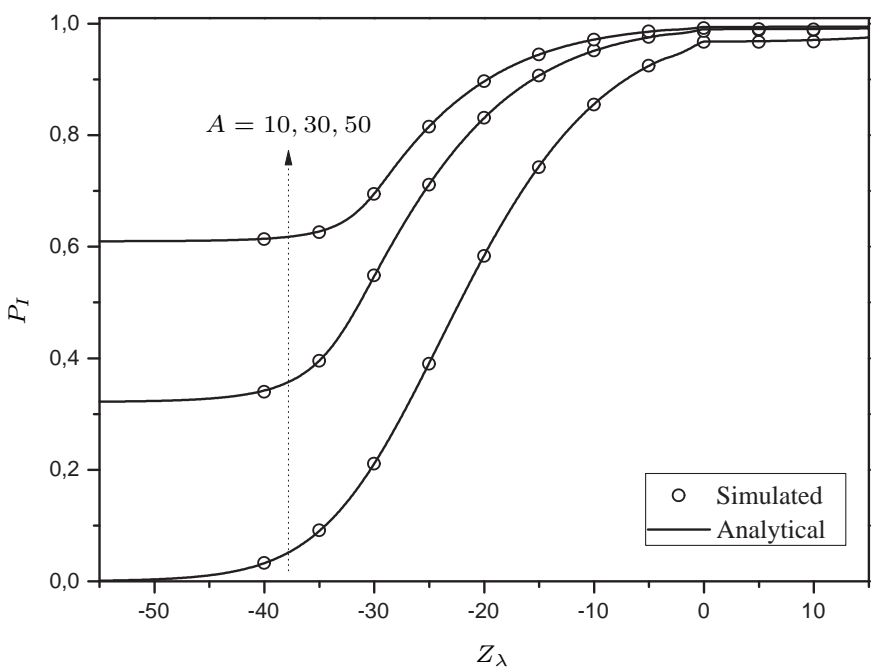

Fig. 4. Outage probability as a function of the SINR threshold $Z_{\lambda}$ considering three possible routes between source and destination nodes.

TABLE II

ANALYTICAL AND SIMULATED VALUES OF OUTAGE PROBABILITY WITH UNIFORM TRAFFIC (30Erl) AND DIFFERENT VALUES OF $Z_{\lambda}$.

\begin{tabular}{|c|cc|cc|cc|}
\hline \multirow{2}{*}{ Pair } & \multicolumn{2}{|c|}{$Z_{\lambda}=-40 d B$} & \multicolumn{2}{c|}{$Z_{\lambda}=-30 d B$} & \multicolumn{2}{c|}{$Z_{\lambda}=-20 d B$} \\
\cline { 2 - 7 } & An. & Sim. & An. & Sim. & An. & Sim. \\
\hline \hline N1-N2 & 0.7345 & 0.7343 & 0.7075 & 0.7079 & 0.7077 & 0.7079 \\
\hline N1-N3 & 0.5951 & 0.5933 & 0.6961 & 0.6963 & 0.8372 & 0.8366 \\
\hline N1-N4 & 0.3213 & 0.3206 & 0.4775 & 0.4777 & 0.6859 & 0.6855 \\
\hline N2-N3 & 0.3461 & 0.3453 & 0.4069 & 0.4057 & 0.6816 & 0.6805 \\
\hline N2-N4 & 0.5140 & 0.5138 & 0.6099 & 0.6096 & 0.8333 & 0.8333 \\
\hline N3-N4 & 0.1728 & 0.1720 & 0.3827 & 0.3820 & 0.6840 & 0.6838 \\
\hline
\end{tabular}

any given time. This can be observed as a discontinuity around $0 d \mathrm{~B}$, present in all curves. From this point on, the network becomes limited by interference, and at most one call, which occupies only one link, can be admitted into the network.

Next, we present a scenario in which the network is asymmetric, defined as a network with different capacities per link. In particular, $C 1=10, C 2=20, C 3=30$ and $C 4=40$. The values of the outage probability for each source-destination pair obtained from the analytical method and from the simulation, for different values of interference margin $Z_{\lambda}$ specified at each column, are shown in Table II. Again an excellent agreement between simulated and theoretical results is attained. Many other scenarios have been tried and our formulations have proved useful.

\section{Conclusions}

In this paper, we have presented an improved analytical method to compute the outage probability in wireless multihop networks. The evaluation of the outage probability is an important step in the network dimensioning process. We focused on the following aspects: multihop traffic, additive noise, joint interference and routing, all combined. An exact formulation of the probability of interference for at least one 
call considering additive Gaussian noise was proposed based on the Joint Outage Probability. This formulation was used to derive a reduced load approximation which considers the traffic of all source-destination pairs of nodes and the routing probabilities to calculate outage probabilities for each link and, consequently, for each route and then for each pair of nodes. In order to validate the method, a multihop network with multiple routes was simulated. Analytical and simulation results, as shown here, are, in practice, indistinguishable from each other.

\section{REFERENCES}

[1] J. Li, C. Blake, D. de Couto, H. Lee and R. Morris, "Capacity of Ad Hoc Wireless Networks," in 7th Annual ACM/IEEE International Conference on Mobile Computing and Networking (MobiCom), 2001, pp. 61-69.

[2] P. Gupta and P. R. Kumar, "The Capacity of Wireless Networks," IEEE Transactions on Information Theory, vol. 46, no. 2, pp. 388-404, Mar. 2000.

[3] A. Futernik, A. M. Haimovich and S. Papavassiliou, "An Analytical Model for Measuring QoS in Ad-Hoc Wireless Networks," in IEEE Global Telecommunications Conference GLOBECOM '03, Dec. 2003, pp. 216-220.

[4] Y. Gugrajah and F. Takawira, "Analytical Model for Evaluating Blocking Probability in Wireless Ad Hoc Networks," in 6th Africon Conference in Africa IEEE AFRICON, Oct. 2002, pp. 277-282.

[5] A. A. M. de Medeiros and M. D. Yacoub, "An Analytical Approach for Dimensioning Wireless Multihop Networks," in IEEE Global Telecommunications Conference, 2007. GLOBECOM '07, Nov. 2007, pp. 4687-4691.

[6] Flavio P. Calmon and Michel Daoud Yacoub, "A General Exact Formulation for the Outage Probability in Interference-Limited Systems," in IEEE Global Telecommunications Conference, 2008. GLOBECOM '08, Nov. 2008, pp. 1-5.

[7] A. Kumar, D. Manjunath and J. Kuri, Communication Networking: An Analytical Approach. Morgan Kaufman, 2004. 